\title{
An overview of intracranial aneurysms
}

\author{
Alexander Keedy*
}

\begin{abstract}
Intracranial aneurysms are relatively common, with a prevalence of approximately $4 \%$. Unruptured aneurysms may cause symptoms mainly due to a mass effect, but the real danger is when an aneurysm ruptures, leading to a subarachnoid hemorrhage. Most aneurysms are asymptomatic and will not rupture, but they grow unpredictably and even small aneurysms carry a risk of rupture. Intracranial aneurysms are diagnosed and monitored with imaging including intra-arterial digital subtraction angiography, computed tomography angiography, magnetic resonance angiography, and recently transcranial Doppler ultrasonograpy has been proposed as a potential modality. Treatment options include observation, endovascular coiling, and surgical clipping. This paper will review the epidemiology, pathogenesis, clinical presentation, diagnosis, natural history, and management of unruptured saccular intracranial aneurysms.
\end{abstract}

\section{INTRODUCTION}

Aneurysms of the cerebral vasculature are relatively common. A recent systematic review collecting data from many countries reported a prevalence of $0.4 \%$ and $3.6 \%$ in retrospective and prospective autopsy studies, respectively, and $3.7 \%$ and $6.0 \%$ in retrospective and prospective angiographic studies, respectively (1). The angiographic studies likely overestimate the true prevalence due to a selection bias, whereas the retrospective autopsy studies likely underestimate the true prevalence due to an inability to review the original material. Eighty-five percent of saccular aneurysms of the cerebral vasculature occur in the circle of Willis (2). Multiple aneurysms are seen in $30 \%$ of patients. Most are small and asymptomatic, but each year, approximately 30,000 people in the United States suffer a rupture, peaking in the sixth decade (3). When an intracranial aneurysm ruptures, it may bleed into the brain parenchyma resulting in a parenchymal hemorrhage, or more often, it will bleed into the subarachnoid space, resulting in a subarachnoid

*To whom correspondence should be addressed: Alexander Keedy, akeedy@uci.edu hemorrhage (SAH). A SAH is a catastrophic event with a mortality rate of $25 \%$ to $50 \%$. Permanent disability occurs in nearly $50 \%$ of the survivors, thus, only approximately one-third of patients who suffer a SAH have a positive outcome (3).

\section{RISK FACTORS}

There are many risk factors for the development of intracranial aneurysms, both inherited and acquired. Females are more prone to aneurysm rupture, with SAH 1.6 times more common in women. The prevalence of aneurysms is increased in certain genetic diseases; the classic example is autosomal dominant polycystic kidney disease (ADPKD), but other diseases such as Ehlers-Danlos syndrome, neurofibromatosis, and a1antitrypsin deficiency also demonstrate a link. In ADPKD, $10 \%$ to $15 \%$ of patients develop intracranial aneurysms. Marfan's Syndrome was once thought to be linked to intracranial aneurysm formation, but recent evidence suggests that this may not be true. Aneurysms also run in families in the absence of an identified genetic disorder, with a prevalence of $7 \%$ to $20 \%$ in first or second degree relatives of patients who have suffered a SAH (2, 3). The acquired risk factors are listed in Table 1. 
Table 1. Acquired risk factors for intracranial aneurysms (4).

Increasing age

Hypertension

Smoking

Alcohol abuse

Estrogen deficiency

Hypercholesterolemia

Carotid artery stenosis

\section{PATHOGENESIS}

There are four main types of intracranial aneurysms: saccular, fusiform, dissecting, and micotic type. The saccular type accounts for $90 \%$ of intracranial aneurysms, thus, it will be the focus of this review. Saccular aneurysms are a result of abberations to the normal arterial structure, which consists of the tunica intima adjacent to the lumen of the vessel, the tunica media (the muscular middle layer), and the tunica adventitia (the outer layer composed mainly of connective tissue). The internal elastic lamina delimits the tunica intima from the tunica media, and the external elastic lamina delimits the tunica media from the tunica adventitia. Saccular aneurysms occur when there is collagen deficiency in the internal elastic lamina and breakdown of the tunica media. An outpouching, consisting of only tunica intima and adventitia, protrudes through the defect in the internal elastic lamina and tunica media to produce the aneurismal sac $(5,6)$. The impaired integrity of the wall may be due to congenital weakness or absence of the tunica media or adventitia, degenerative alterations of the internal elastic lamina (from hypertension, turbulent flow, or atherosclerotic deposits in the wall), or both of these factors combined (2). Low collagen and elevated plasma elastase has been observed in patients with aneurysms, suggesting that vascular remodeling involving collagen and elastin plays an important role (4).

Eighty-five percent of saccular aneurysms arise from the arteries of the circle of Willis. The most frequent location is the anterior communicating artery (35\%), followed by the internal carotid artery (30\%-including the carotid artery itself, the posterior communicating artery, and the ophthalmic artery), the middle cerebral artery (22\%), and finally, the posterior circulation sites, most commonly the basilar artery tip. See Figure 1 for a scheme of the circle of Willis. Multiple aneurysms are found in approximately $30 \%$ of patients (2).

\section{CLINICAL PRESENTATION}

The symptoms of SAH result from blood spilling into the cerebrospinal fluid (CSF) and the subsequent increased intracranial pressure and breakdown of blood products. Characteristic symptoms include: "the worst headache of my life," nausea and vomiting, loss of consciousness, neck stiffness, and seizures $(7,8)$. The clinical manifestations of unruptured aneurysms, however, are much more subtle. Only $10-15 \%$ of intracranial aneurysms are symptomatic $(9,10)$, with the majority being identified incidentally during evaluation for other conditions. When present, the symptoms are primarily due to the mass effect of a large aneurysm, or possibly from minimal leakage of blood which irritates the meninges, though not enough to be classified as a hemorrhage. These symptoms include headache, unilateral third cranial nerve palsy (from a posterior communicating artery aneurysm), bilateral temporal hemianopsia (from an anterior communication artery aneurysm impinging on the optic chiasm) ischemic cerebrovascular disease, poorly defined spells, and seizures $(4,11)$. These symptoms may be a warning sign of an impending rupture, as $10 \%$ to $43 \%$ of patients with SAH report experiencing a sentinel headache in the 2 months preceding the rupture (12).

Table 2. Comparison of Sensitivity and Specificity of CTA, MRA, and TCD in the diagnosis of intracranial aneurysms (4).

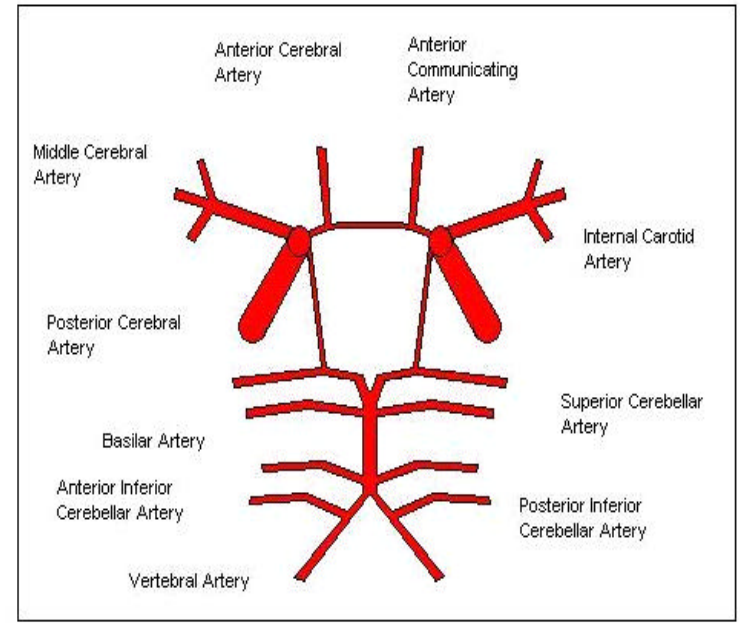

Figure 1. The circle of Willis.

\begin{tabular}{lcc}
\hline Method & Sensitivity & Specificity \\
\hline CTA & 90 & 86 \\
\hline MRA & 87 & 95 \\
\hline TCD & 82 & 95 \\
\hline
\end{tabular}

\section{DIAGNOSIS AND IMAGING FINDINGS}

There are currently three imaging modalities widely used in the diagnosis of intracranial aneurysms: intraarterial digital subtraction angiography (IADSA), computed tomography angiography (CTA) and 
magnetic resonance angiography (MRA). IADSA is similar to conventional angiography in that a catheter is advanced in the arterial system to the point of interest, and radio-opaque contrast material is injected while images are acquired. The contrast fills the lumen of the arteries; thus, the vessel anatomy is visualized on the image. In conventional angiography, serial $x$-ray films are captured, while in IADSA, serial digital images are obtained and stored on a computer. An initial image acquired before contrast injection is subtracted from the post-contrast images. The resultant image displays dark vessels against a blank background (see Figure 2). This technique allows greater contrast resolution (the areas with contrast are more obvious), but decreased spatial resolution (because digitally acquired images have a lower resolution than films) when compared with conventional angiography.

CTA is another technique of vascular imaging which involves obtaining a normal CT scan while intravenous contrast material is injected. The contrast material is radio-opaque, so it appears white on the $\mathrm{CT}$ image. The serial axial slices enhanced with contrast are analyzed by a computer program that forms a three-dimensional reconstruction of the vascular anatomy. The resultant image is a dynamic model that can be rotated in order to view the image from multiple perspectives (see Figure $3)$.

Table 3. The morbidity, mortality, and rebleeding rate of intervention for intracranial aneurysms $(3,4,23,28)$

\begin{tabular}{lcc}
\hline Outcome & Intravascular Coiling (\%) & Surgical Clipping (\%) \\
\hline Mortality & $1.0-1.1$ & $2.6-3.8$ \\
\hline Morbidity & $3.7-4.0$ & $10.9-12.1$ \\
\hline Rebleeding & 2.6 & $0-0.9$ \\
\hline
\end{tabular}

Similar to CTA, MRA is a technique which uses serial axial MRI images to form a three-dimensional representation of the vascular anatomy. Unlike CTA, however, MRA does not require the use of intravenous contrast material. This is because the signal obtained in magnetic resonance imaging depends on the magnetic properties of the area being imaged. A magnetic pulse aligns all the protons in a certain area, and measuring the amount of time necessary for those protons to return to their pre-magnetization state generates the signal which produces the MR image. With a moving substance such as blood, the protons are aligned during the magnetic pulse, but by the time the signal is collected, the aligned protons have moved out of the area which is being imaged, and new 'non-magnetized' protons have taken their place. Because these new protons have not been magnetized, a signal is not generated and the blood vessel lumen appears as a 'signal void' on the image (this is an oversimplified explanation). The lack of signal distinguishes the vessels from the surroundings (see Figure 4). In some cases, a gadolinium contrast material may be used to provide better imaging of the vessels. The advantage of MRA is that it can be used in patients who cannot tolerate the iodine based contrast used in IADSA and CTA, such as patients with allergic reactions or with renal failure.

The gold standard for diagnosis of intracranial aneurysms is currently IADSA, but a diagnosis can also be provided by CTA and MRA. The contrast provided during IADSA causes the aneurysm to appear on fluoroscopy as a radio opaque, smooth margined, saccular out-pouching of the cerebral vasculature (see Figure 2) (13). A thrombus within the aneurismal sac will be visualized as an interruption to the rounded margin because contrast material will not penetrate the clotted, stationary blood. Such a thrombus will be better delineated with CTA or MRA than IADSA, as these former modalities image the vessel wall and surroundings, not only the lumen of the vessel. On CTA, if large enough, an aneurysm will appear as a rounded, spherical mass with attenuation (whiteness) equal to that seen in the large vessels. CT may also depict calcifications in the wall of the aneurysm, or the presence of thrombus within the lumen which will not enhance with contrast medium; these characteristics are seen more frequently in giant aneurysms $(>25 \mathrm{~mm})(14)$. On MRA, the lumen of the aneurysm will appear as a flow void, meaning that it does produce a magnetic signal because blood flow has slowed due to the larger cross-sectional area of the aneurysms compared to the nearby normal vasculature. Areas of high signal intensity surrounding the flow void area of the aneurysm on MRA likely represent a rupture with subsequent hemorrhage: the high signal pattern is produced by stagnant blood (14).

As mentioned above, the gold-standard for diagnosis of cerebral aneurysms is IADSA because it remains the test with the highest spatial resolution. But some cases are not adequately visualized with IADSA alone, as single projections do not provide a sense of volume, and it may fail to detect an aneurysm if the lesion overlaps with nearby vessels. These concerns can be addressed by using $3 \mathrm{D}$ rotational angiography, which images the cerebral vasculature on many views. However, IADSA is costly and invasive with a $2-4 \%$ transient complication rate and about $0.5 \%$ of patients undergoing catheterization are left with permanent neurological complications $(15,16,17)$. Typical complications include amaurosis fugax, hemiparesis, 
confusion, muscle spasm, and aphasia. Because of these considerations and the knowledge that CTA and MRA are also effective tests, there is some debate over the best way to diagnose an aneurysm $(18,19,20)$. Transcranial Doppler sonography has also been proposed as a method to evaluate the presence of aneurysms (21). See Table 2 for the sensitivity and specificity described for the various techniques. However, it should be noted that these numbers are based on studies with small sample sizes, and other methodological flaws, and at this time, the available data do not suggest that one modality is clearly superior to the others (3). It is also necessary to mention that the values found in Table 2 are highly dependent on the size of the aneurysm. If the aneurysm is $<3 \mathrm{~mm}$, the sensitivity of MRA plummets to $38 \%$, and the sensitivity of CTA drops to $61 \%$ (2). Because the technology in radiological imaging is improving rapidly with increases in resolution of all modalities, it is expected that the values listed in Table 2 will improve in the coming years. Illustrating this point, a recent study demonstrated CTA and IADSA to both have a sensitivity of $97 \%$ and a specificity of $100 \%$ when evaluating middle cerebral artery aneurysms. In $76 \%$ of the cases CTA was able to provide information not available on IADSA, suggesting that CTA, in its current state, may be superior to the "gold-standard" (22).

\section{NATURAL HISTORY OF UNRUPTURED INTRACRNIAL ANEURYSMS}

There is a great deal of controversy surrounding the natural history of unruptured aneurysms. It is such a hot-topic because knowing the likely course of aneurysms will play a pivotal role in determining the appropriate management. Unlike the more predictable course of abdominal aortic aneurysms, where the lesion grows in size and rarely ruptures before it reaches the threshold diameter of $5.0 \mathrm{~cm}$, the data for intracranial aneurysms are much less clear.

Prior to 1998, the data available estimated the rupture rate for aneurysms to be 1 to $2.5 \%$ per year. This value indicates that over many years, the risk of rupture is very significant, and therefore, surgical or endovascular treatment is appropriate in patients with incidental aneurysms (23). However, in 1998, data published from a study called the International Study of Unruptured Intracranial Aneurysms (ISUIA) challenged many of the beliefs about the natural history of aneurysms. This study had both retrospective and prospective parts. The retrospective part found that in patients whose aneurysm measured $<10 \mathrm{~mm}$ and without a prior history of SAH, the risk of rupture was $0.05 \%$ per year and the risk of rupture for aneurysms $>10 \mathrm{~mm}$ was $1 \%$ per year (24). These data indicate that the rupture risk of aneurysms may be much lower than what was previously believed. This changes the recommendation for intervention, because if the data from the ISUIA are correct, the risk of intervention outweighs the risk of rupture in small aneurysms. The data from this study also indicate that the size of the aneurysm is the best predictor of future rupture, with $10 \mathrm{~mm}$ appearing to be an appropriate cut-off point between small and large aneurysms. The location of the lesion also had an independent effect on the risk of rupture, with aneurysms in the posterior circulation having a higher risk (24). In 2003, data from the prospective segment of the study were published, and indicated the five-year rupture risk is $0 \%$ and $2.5 \%$ for small aneurysms in the anterior and posterior circulation, respectively. This portion of the study also suggested that the upper limit demarcation of a small aneurysm (thus, low of rupture) is $7 \mathrm{~mm}$, not $10 \mathrm{~mm}$ as suggested by the retrospective segment of the ISUIA (25).

There are many criticisms of the ISUIA; a few of which are subsequently discussed. First, the patients with unstable aneurysms were selected out of the retrospective part of the study because they would have been treated upon the discovery of the lesion, and not included in the data. Second, cavernous carotid aneurysms were included in the study group, but these lesions generally do not produce SAH. Third, the annual incidence of SAH in the United States is about 30,000 , which can be calculated to a rupture rate of at least $1 \%$ per year (23). These points suggest that the rupture rate suggested in the ISUIA may be artificially low.

Despite these criticisms, this was indeed a landmark study, and the clinical guidelines are based largely on the data from this trial. The current recommendations are observation, rather than intervention, in patients with incidental aneurysms $<10 \mathrm{~mm}$. Exceptions include patients with growing lesions and those approaching $10 \mathrm{~mm}$, lesions with irregular morphological and hemodynamic features, and patients with multiple first or second degree relatives with a history of aneurismal SAH.

\section{MANAGEMENT}

The appropriate management for unruptured intracranial aneurysms does not have a clear evidencebased strategy. The risks of rupture must be weighed against the risks associated with intervention. Three main treatment options are available to the patient: observation, endovascular therapy, and surgical therapy.

In light of the ISUIA data, observation is a viable option in many cases. If this management is selected, it is important for the treating physician to be aware that aneurysms may grow unpredictably, so it is necessary to 
serially monitor the aneurysm and watch for new-onset symptoms related to the aneurysm. Serial monitoring can be accomplished non-invasively with MRA, CTA, or possibly transcranial Doppler ultrasonography (see section on diagnosis). The parameters suggesting that a patient should not undergo intervention include: lack of symptoms, aneurysm size $<7 \mathrm{~mm}$, lesion in the anterior circulation, age older than 64 years, and no personal or family history of SAH. Similarly, patients younger than 50 years with symptomatic aneurysms $>25 \mathrm{~mm}$ located in the posterior circulation and a personal or family history of SAH should undergo intervention (4). Of course, most cases fall somewhere in between these two extremes and the appropriate management is not obvious.

Approved by the FDA in 1991, endovascular treatment consists of guiding a catheter from the femoral artery to the cerebral vasculature via the ICA or vertebral artery, depending on the location of the aneurysm. The procedure is guided by fluoroscopy, and when the catheter has reached the aneurysm, several soft platinum coils are deployed in the lumen of the lesion. These coils completely fill the lumen and induce the formation of a thrombus to occlude the aneurysm, preventing future rupture (4). A wide neck and large size of the aneurysm make the procedure more difficult with poorer results. This procedure appears to be safe relative to surgical treatment, and it is able to treat lesions that are difficult to approach surgically, but there are questions regarding the durability of the endovascular technique. In most studies, complete occlusion is achieved in $80 \%$ to $90 \%$ of patients. At post-treatment follow-up, however, small neck remnants are common, and some degree of thrombus recanalization is observed in $50 \%$ of all patients and up to $90 \%$ of patients with giant aneurysms. Both neck remnants and recanalization are associated with a risk of rupture, and $20 \%$ of patients may require more than one coiling procedure (23). Contrast enhanced transcranial Doppler sonography has been shown by a small study to be sensitive and specific (100\% and $97 \%$, respectively) in the detection of clinically relevant residual flow in the follow-up of coil-embolized aneurysms (26). IADSA is effective in visualizing treatment failures with residual necks or complete recanalization, but 3D MR appears to be more effective in evaluating "partial treatments" (27), such as incomplete recanalization, which may prove to be useful in detecting treatment failures before they have the potential to hemorrhage.

Surgical clipping has been used for the treatment of intracranial aneurysms for longer than 40 years. This procedure involves placing a surgical clip at the junction of the healthy artery and the neck of the aneurysm. This treatment is very effective, demonstrated by annual risks of rupture following clipping reported from $0 \%$ to $0.9 \%(4,28)$. The disadvantage is the invasive nature of the procedure, which is associated with a higher risk of complications, and as with any surgery, elderly patients are less able to tolerate the trauma of the procedure. See Table 3 for a comparison of the two treatment interventions.

\section{SUMMARY}

Intracranial aneurysms are relatively common,

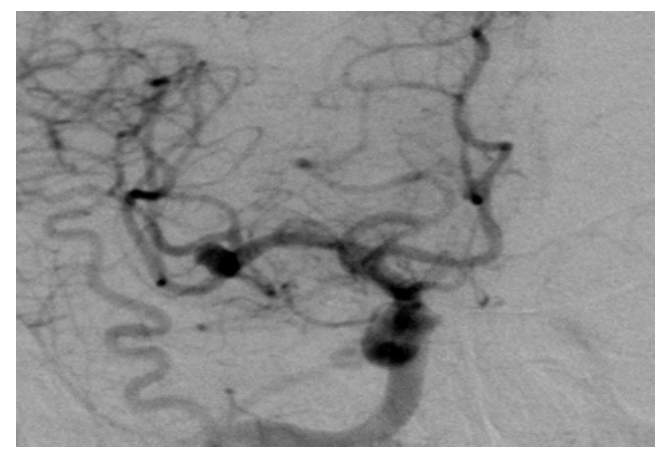

Figure 2. Bilobed aneurysm of the right middle cerebral artery measuring $4 \times 5 \mathrm{~mm}$.

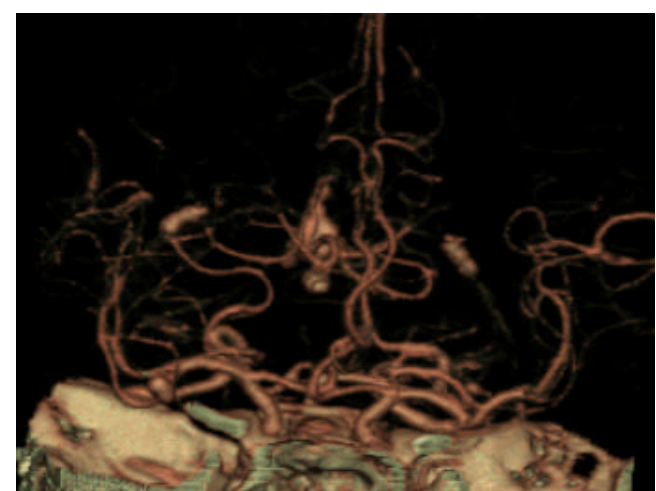

Figure 3. Computed tomography (CT) angiography of the same aneurysm depicted in Figure 2.

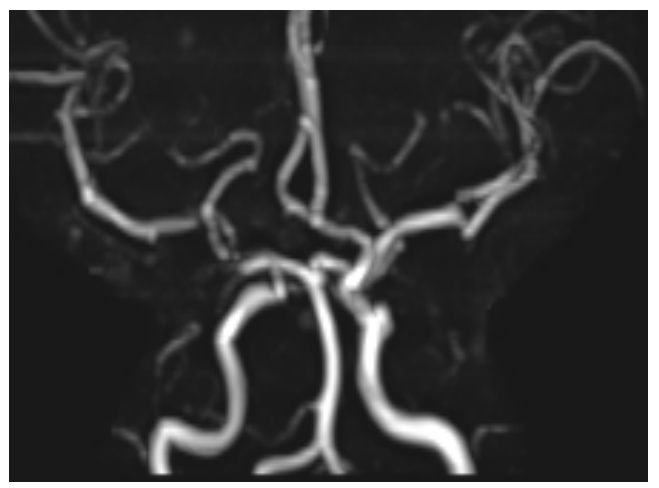

Figure 4. Magnetic resonance (MR) angiogram depicting normal cerebral vasculature 
occurring in approximately $4 \%$ of the population. Most of these aneurysms are asymptomatic, and they carry a small but real risk of rupture, resulting in a subarachnoid hemorrhage. IADSA is the gold-standard for diagnosis of intracranial aneurysms, but CTA, MRA, and transcranial Doppler sonography are also effective diagnostic tests. These non-invasive imaging modalities are more appropriate for serially monitoring aneurysms because of the risks associated with invasive angiography. The natural history of intracranial aneurysms is still being explored, but our current understanding suggests that the annual risk of rupture is $1 \%$ or less. Large, irregularly shaped lesions arising from the posterior circulation are at an increased risk for rupture. The management strategies consist of observation, intravascular coiling, and surgical clipping .

\section{REFERENCES}

1. Rinkel GJ, Djibuti M, Algra A, et al. Prevalence and risk of rupture of intracranial aneurysms: a systematic review. Stroke 1998; 29: 251-6.

2. Gasparotti R, Liserre R. Intracranial aneurysms. Eur Radiol 2005; 15: 441-7.

3. Wardlaw JM, White PM. The detection and management of unruptured intracranial aneurysms. Brain 2000; 123: 205-21.

4. Wagner M, Stenger K. Unruptured intracranial aneurysms: using evidence and outcomes to guide patient teaching. Crit Care Nurs Q 2005; 28: 341-54.

5. Austin G, Fisher S, Dickson D, et al. The significance of the extracellular matrix in intracranial aneurysms. Ann Clin Lab Sci 1993; 23: 97-105.

6. Stehbens WE, Delahunt B, Hilless AD. Early berry aneurysm formation in Marfan's syndrome. Surg Neurol 1989; 31: 200-2.

7. Liebenberg WA, Worth R, Firth GB, et al. Aneurysmal subarachnoid haemorrhage: guidance in making the correct diagnosis Postgrad Med J 2005; 81: 470-3.

8. Gorelick PB, Hier DB, Caplan LR, et al. Headache in acute cerebrovascular disease. Neurology 1986; 36: 1445-50.

9. Friedman JA, Piepgras DG, Pichelmann MA, et al. Small cerebral aneurysms presenting with symptoms other than rupture. Neurology 2001; 57: 1212-6.

10. Wiebers DO, Whisnant JP, Huston J 3rd, et al. International Study of Unruptured Intracranial Aneurysms Investigators. Unruptured intracranial aneurysms: natural history, clinical outcome, and risks of surgical and endovascular treatment. Lancet 2003; 362: 103-10.

11. Rowley HA. Cerebrovascular Disease. In: Brant W, Helms C. Fundamentals of Diagnostic Radiology. Baltimore, MD: Williams \& Wilkins; 1994.

12. Polmear A. Sentinel headaches in aneurysmal subarachnoid haemorrhage: what is the true incidence? A systematic review. Cephalalgia 2003; 23: 935-41.

13. Novelline R. The Central Nervous System. In: Novelline R. Squire's Fundamentals of Radiology, 5th ed. Cambridge, MA: Harvard University Press; 1997.
14. Jager R, Saunders D, Murray A. Cranial and intracranial pathology (2): cerebrovascular disease and nontraumatic intracranial hemorrhage. In: Grainger R, Allison D, Adam A, Dixon A. Diagnostic Radiology: A Textbook of Medical Imaging, 4th ed. London, England: Harcourt Publishers Limited; 2001.

15. Leffers AM, Wagner A. Neurologic complications of cerebral angiography. A retrospective study of complication rate and patient risk factors. Acta Radiol 2000; 41: 204-10.

16. Waugh JR, Sacharias N. Arteriographic complications in the DSA era. Radiology 1992; 182: 243-6.

17. Warnock NG, Gandhi MR, Bergvall U, Powell T. Complications of intraarterial digital subtraction angiography in patients investigated for cerebral vascular disease. Br J Radiol 1993; 66 (790): 855-8.

18. Tipper G, U-King-Im JM, Price SJ, et al. Detection and evaluation of intracranial aneurysms with 16-row multislice CT angiography. Clin Radiol 2005; 60: 565-72.

19. Kouskouras C, Charitanti A, Giavroglou C, et al. Intracranial aneurysms: evaluation using CTA and MRA. Correlation with DSA and intraoperative findings. Neuroradiology 2004; 46: 842-50.

20. Chappell ET, Moure FC, Good MC. Comparison of computed tomographic angiography with digital subtraction angiography in the diagnosis of cerebral aneurysms: a meta-analysis. Neurosurgery 2003; 52: 624-3.

21. Turner CL, Kirkpatrick PJ. Detection of intracranial aneurysms with unenhanced and echo contrast enhanced transcranial power Doppler. J Neurol Neurosurg Psychiatry 2000; 68: 489-95.

22. Villablanca JP, Hooshi P, Martin N, et al. Three-dimensional helical computerized tomography angiography in the diagnosis, characterization, and management of middle cerebral artery aneurysms: comparison with conventional angiography and intraoperative findings. J Neurosurg 2002; 97: 1322-32.

23. Tummala RP, Baskaya MK, Heros RC. Contemporary management of incidental intracranial aneurysms. Neurosurg Focus 2005; 18: 9:1-7.

24. The International Study of Unruptured Intracranial Aneurysms Investigators: Unruptured Intracranial Aneurysms-risk of rupture and risk of surgical intervention. N Engl J Med 1998; 339: 1725-33.

25. Turner CL, Higgins JN, Kirkpatrick PJ. Assessment of transcranial color-coded duplex sonography for the surveillance of intracranial aneurysms treated with Guglielmi detachable coils. Neurosurgery 2003; 53: 866-71.

26. Costalat V, Lebars E, Sarry L, et al. In vitro evaluation of 2Ddigital subtraction angiography versus 3D-time-of-flight in assessment of intracranial cerebral aneurysm filling after endovascular therapy. Am J Neuroradiol 2006; 27: 177-84.

27. Molyneux A, Kerr R, Stratton I, et al; International Subarachnoid Aneurysm Trial (ISAT) Collaborative Group. International Subarachnoid Aneurysm Trial (ISAT) of neurosurgical clipping versus endovascular coiling in 2143 patients with ruptured intracranial aneurysms: a randomised trial. Lancet 2002; 360: 1267-74.

Alexander Keedy completed his undergraduate training at Santa Clara University and is currently a third year medical student at University of California, Irvine (UCI). His research interests include the diagnostic accuracy of CT in cirrhosis and the clinical utility and applications of 3D CT reconstructions. He will postpone his fourth year of medical school for one year in order to work on a project to determine the efficacy of teaching anatomy and radiology with three dimensional displays at UCSF. 